dated wood, in order to provide a calibration of the radiocarbon time scale that could be used for converting conventional radiocarbon dates to calendaric dates.

It was disappointing that the conversion graphs and tables produced by the two methods did not agree sufficiently well for the conference to advise their adoption for calibration purposes. This adoption must be deferred until at least the next international conference on dating, for new data will make little difference to the curves for some time. The causes of carbon-14 variations in terms of geomagnetic and heliomagnetic cycles as well as climatic changes at the end of the last glaciation were sought as explanations by Professor A. Fairhall (University of Washington), Dr E. Ralph (University of Pennsylvania) and Dr M. Baxter (University of Glasgow). Dr Baxter warned that fluctuations induced by Sun spots could introduce uncertainties of up to 120 years in the dating of short lived materials such as grain, straw, seeds, single tree rings, twigs or egg shells.

Professor G. Östlund (University of Miami) introduced the GEOSECS oceanic programme, the purpose of which is the detailed measurement of the chemical constituents of water along sections from the Arctic to Antarctic in the Pacific and Atlantic oceans from surface to bottom. These measurements will provide a set of physical and chemical data that can be used for quantitative studies of ocean mixing and ocean productivity. They will also provide a base line for levels of pollutants and of fission and waste products being added to the sea. One of the most important sections of the programme is the measurement of carbon-14, because its half life is comparable with the time scale of mixing processes occurring in the deep ocean. Special carbon-14 laboratories are being established at Miami and Seattle (Professor M. Stuiver) to deal with the anticipated 1,200 samples each of 200 litres of water.

The nuclear bombs exploded since 1955 have doubled the radioactive carbon dioxide in the troposphere, and large doses have been injected into the lower atmosphere through the tropopause gap. All terrestrial tissues have reacted naturally to this and compounds involved in the usual processes of photosynthesis and feeding have had a fraction of all their carbon positions labelled. Mr H. S. Jansen (Institute of Nuclear Sciences, Lower Hutt) pointed out that there was now an opportunity for measuring residence times (that is, the mean time before a carbon atom residing in a tissue is replaced by atmospheric carbon) in such a way that could be expected to reflect biochemical processes in the animal or plant tissue without conducting experiments which might alter the process. He regretted the fact that the opportunity was being lost by the workers in the life sciences because the activity was at present falling by 1.5 per cent each year. He assumed that this lack of use of "bomb carbon" is probably attributable to inadequate communications between disciplines.
In an attempt to redress this, Mr Jansen has compiled families of curves for various latitudes and different years of origin or birth of the tissue. He has plotted $\triangle{ }^{14} \mathrm{C}$, relative radiocarbon enrichment of the tissue against calendar years so that, at a glance, a researcher can read off the rate of replacement from the curves. Other experiments can be planned with their aid.

\title{
Synthesis of Myeloma Light Chain DNA
}

THE biological role of tumour virus reverse transcriptase is apparently the synthesis of a DNA provirus during the establishment of infection, but be that as it may this enzyme in the hands of biochemists is an invaluable tool for the synthesis of DNA chains which can be labelled to a high specific activity and which are complementary to specific cellular RNA molecules. Single stranded DNA molecules complementary to at least part of globin messenger RNA molecules have recently been synthesized and partially purified, and in next Wednesday's Nature New Biology (February 7) Aviv, Packman, Swan, Ross and Leder report the synthesis of a DNA complementary to about 40 per cent of a mouse myeloma light chain messenger RNA.

Aviv et al. purified reverse transcriptase from avian myeloblastosis virus and partially purified light chain messenger RNA, which in a cell free system stimulates immunoglobulin light chain synthesis, from mouse MOPC 41 tumour cells. Using oligo (dT) as a specific primer of reverse transcription, the oligo (dT) presumably hydrogen bonds to the poly (A) residues at the $3^{\prime}$ terminus of the mRNA molecules, they synthesized a 5S DNA which contains about 325 nucleotides. Hybridization experiments with this DNA and polysomal RNAs from MOPC 41 cells and another line of myeloma cells, MOPC 315 , indicate that the product of reverse transcription is heterogeneous.

In addition to a major species of DNA complementary to the light chain mRNA DNAs complementary to RNAs common to both MOPC 41 and MOPC 315 cells are made. As Aviv et al. point out, however, it should be possible to purify the major DNA species and use it to discover the number of myeloma light chain genes in these mouse MOPC 41 cells.

\section{Two New Infrared Sources Discovered}

STudies of astronomical sources in the infrared continue to be carried out by the group at University College, London. Telescopes carried on balloon flights from Texas last September scanned known objects, and also resulted in the discovery of two new

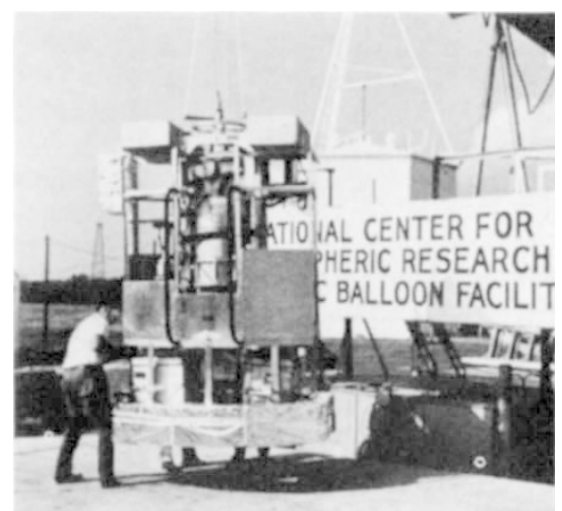

The UCL balloon-borne infrared telescope being rigged at NCAR, Texas, shortly before launch. sources, not previously detected in the infrared. These objects, RCW 117 and DR 15, are both HII regions, and were previously known by their radio emission.

Details of the observations are reported by Emerson, Jennings and Moorwood in next Monday's Nature Physical Science (February 5). RCW 117 appears as a compact infrared source, smaller than the 4 arc min beamwidth of the detector. It seems that it is a compact HII region, whereas the other object discussed in this work, DR 15 , is not particularly compact. The new observations at infrared frequencies have made it possible to test the theory that much radiation is produced by the degradation of ultraviolet photons by dust grains. The data suggest that the amount of ultraviolet energy required could be supplied by an underlying young $\mathrm{O}$ star within the HII region, but that the radiation would then have to be coupled very efficiently to the dust. 